\section{Case Reports in Dermatology}

Case Rep Dermatol 2021;13:1-6

This article is licensed under the Creative Commons Attribution-NonCommercial 4.0 International License (CC BY-NC) (http://www.karger.com/Services/OpenAccessLicense). Usage and distribution for commercial purposes requires written permission.

\title{
Intralymphatic Histiocytosis: An Unusual Presentation
}

\author{
Timothy J. Blackwell ${ }^{\mathrm{a}} \quad$ Zachary Ingersoll ${ }^{\mathrm{b}} \quad$ Martin Blackwell ${ }^{\mathrm{b}}$ \\ aRowan School of Osteopathic Medicine, Stratford, NJ, USA; ${ }^{b}$ Dermatology, Hackensack \\ Meridian Health Palisades Medical Center, North Bergen, NJ, USA
}

\section{Keywords}

Intralymphatic histiocytosis · Granulomatous · Autoimmune · Psoriasis

\begin{abstract}
Intralymphatic histiocytosis (ILH) is a rare cutaneous condition initially described in 1994 by O'Grady et al. It often appears as a red to violaceous, livedoid patch or plaque usually on the extremities. We present a 71 -year-old female with a history of psoriasis, 50 pack years smoking and recent Legionnaires disease who came to us complaining of a red to violaceous, blanching, edematous, mildly tender lesion covering the left lower lip and extending to the chin and anterior neck. After multiple biopsies, ILH was confirmed and the patient was initially started on tacrolimus $0.1 \%$ ointment b.i.d., but there was no response. Then, she was started on oral pentoxifylline and intermittent topical steroids, as well as continuing the topical tacrolimus. There was again no response, so now she is taking a TNF-a inhibitor as it appears to be a granulomatous process. These ILH cases are very rare and there is limited literature that describes one treatment as a cure. Treatment of ILH is very difficult, but several different therapies have been reported with varying success. If the disease is secondary to an underlying inflammatory disease or malignancy, then treatment of the primary disorder can lead to resolution of the ILH.




\section{Case Reports in Dermatology}

Case Rep Dermatol 2021;13:1-6

\begin{tabular}{l|l}
\hline DOI: $10.1159 / 000510128$ & (c) 2021 The Author(s). Published by S. Karger AG, Basel
\end{tabular} www.karger.com/cde

Blackwell et al.: Intralymphatic Histiocytosis: An Unusual Presentation

\section{Introduction}

Intralymphatic histiocytosis (ILH) is a rare cutaneous condition initially described in 1994 by O'Grady et al. [1]. It often appears as a red to violaceous, livedoid patch or plaque usually on the extremities. The condition is most commonly seen in association with rheumatoid arthritis. Other associations have been identified such as orthopedic implants, chronic inflammation, and underlying malignancy [2,3]. Interestingly, it has also been described as a histologic feature of granulomatous cheilitis and Melkersson-Rosenthal syndrome (MRS) [4]. We present the case of a 71-year-old female with cutaneous intralymphatic histiocytosis of the lower face with uncertain etiology.

\section{Case Presentation}

A 71-year-old female initially presented to another dermatologist complaining of a bright red, warm and tender plaque on the left lower lip. Her past medical history was significant for 50 pack years of smoking, psoriasis, and a hospitalization 1 year prior from Legionnaire's disease. She was initially treated for a presumed infection without improvement. She was also referred to ENT, who recommended CT of the face and sinuses, which was negative for any significant findings.

Six months after initial presentation to a dermatologist, the patient presented to our clinic with a 7-month history of a red to violaceous, blanching, edematous, mildly tender lesion covering the left lower lip and now extending to the chin and anterior neck (Fig. 1, 2). Intraoral exam was negative for any changes. On this visit, there was concern for possible malignancy, and a 3-mm punch biopsy of involved skin on the left lower cutaneous lip was performed. This was interpreted as having "features most consistent with glomeruloid hemangioma." In light of this pathology report, a workup for POEMS syndrome was initiated, although clinically the lesion did not appear to be consistent with this diagnosis.

Three weeks later, a repeat biopsy was performed and sent to a different laboratory for evaluation. The initial 3-mm punch biopsy was also sent for a second opinion. These two biopsies were found to be most consistent with intravascular/intralymphatic histiocytosis with features of granulomatous vasculitis (Fig. 3, 4). The patient was also sent for a second opinion, and a third and confirmatory biopsy of the left chin again showed intralymphatic histiocytosis. Initial bloodwork showed hemoglobin of 16.6 and hematocrit of 49.7, CEA of 6.4 (ref: 0-4.7), with ANA and ANCA negative. Age-appropriate cancer screening was also recommended and is still pending at this time. The patient had an MRI done which showed asymmetric prominence of the subcutaneous fat over the left face with no abnormal soft tissue mass or lymphadenopathy.

As the diagnosis was not immediately evident, treatment was initiated with tacrolimus $0.1 \%$ ointment b.i.d. While this treatment did not shrink the lesion, it prevented the lesion from growing in size. Once the final diagnosis was made, it was decided to start oral pentoxyfylline and intermittent topical steroids, as well as continuing the topical tacrolimus. The patient was on this treatment regimen for 1 month with no improvement. As this appears to be a granulomatous process, our current plan is to pursue treatment with a TNF-a inhibitor. 


\section{Case Reports in Dermatology}

Case Rep Dermatol 2021;13:1-6

\begin{tabular}{l|l}
\hline DOI: $10.1159 / 000510128$ & (c) 2021 The Author(s). Published by S. Karger AG, Basel
\end{tabular} www.karger.com/cde

Blackwell et al.: Intralymphatic Histiocytosis: An Unusual Presentation

\section{Discussion/Conclusion}

Intralymphatic histiocytosis (ILH) is an extremely rare disease that most often affects elderly women [5]. This disease has an inconsistent presentation ranging from a single erythematous plaque to multiple papules or nodules [6]. The lesions can also be seen as poorly demarcated and irregularly shaped patches or plaques with a livedo-reticularis like pattern [7]. There is a strong association with rheumatoid arthritis with other less common associations such as Rosai-Dorfman disease and orthopedic metal implants. Of the 34 patients in the literature with ILH, 18 patients had rheumatoid arthritis and two additional patients had a positive rheumatoid factor [7]. There is some uncertainty about the pathogenesis of intralymphatic histiocytosis. It is widely accepted that chronic inflammation leads to obstruction and dilation of lymphatic vessels. Lymphostasis leads to poor antigen clearance, constant stimulation of the immune system, and proliferation of histiocytes. This proliferation perpetuates the obstruction as it leads to further adhesion of histiocytes [8].

In a review of 16 cases of ILH, the majority of the lesions were seen on the extremities and breast region $[7,8]$. The most common morphology is a pink to violaceous plaque which is then followed by papules and nodules. One lesion on the upper eyelid in this review was associated with MRS [6]. Another case report described ILH in an 8-year-old boy with MRS of the upper lip [9]. In our case, the patient presented with a warm, tender, erythematous plaque of the lower lip which then spread to the chin and neck. The morphology was consistent with previously reported cases of ILH; however, the location of the lesion, and other histologic features not usually seen in orofacial granulomatosis, made it difficult to establish that specific diagnosis.

One review of 16 case reports of ILH identified some common characteristics of the disease. It was found that the epidermis and papillary dermis were unchanged, but the reticular dermis showed dilated vascular and lymphatic structures. Within these dilated vessels, some retained an empty lumen, while some contained histiocytes both singly and in aggregates [7]. The histiocytes within the lumen of the lymphatic system positively stain for CD31,34 and the vessel itself stains positive for D2-40 [8].

Treatment of ILH is very difficult, but several different therapies have been reported with varying success. If the disease is secondary to an underlying inflammatory disease or malignancy, then treatment of the primary disorder can lead to resolution of the ILH. One example is the treatment of rheumatoid arthritis with infliximab, which lead to resolution of ILH. Another example is the treatment of lung adenocarcinoma with pembrolizumab leading to improvement of ILH [10]. One case that was associated with tonsillitis showed response to antibiotic therapy and tonsillectomy [11]. Other reported treatment options include: electron beam therapy, cyclophosphamide, topical and systemic steroids, amoxicillin and acetylsalicylic acid, pentoxifylline, NSAIDs, excision, pressure bandages, topical tacrolimus, and methotrexate [11].

In summary, this is a very unique case that for now has been diagnosed based on its predominant pathologic feature: intralymphatic histiocytosis. This may represent a case of MRS with unusual pathologic features. However, two separate pathology labs confirmed the diagnosis of intralymphatic histiocytosis. Further discussion and research is needed to more fully understand the pathogenesis and treatment of this uncommon disease. 


\section{Case Reports in Dermatology}

\section{Statement of Ethics}

The authors received written informed consent from this patient to publish this case (including images). The research was conducted ethically in accordance with the World Medical Association Declaration of Helsinki. Information revealing the subject's identity has been avoided and all patients were identified by aliases and not by their real names.

\section{Conflict of Interest Statement}

The authors have no conflicts of interest to declare.

\section{Funding Sources}

No funding sources were used for this project.

\section{Author Contributions}

Timothy J. Blackwell: manuscript contributor, author of discussion and case presentation. Zac Ingersoll: manuscript contributor, author of introduction and case presentation. Martin Blackwell: treating physician, consent of patient, manuscript contributor/author of introduction.

\section{References}

1 O'Grady JT, Shahidullah H, Doherty VR, al-Nafussi A. Intravascular histiocytosis. Histopathology. 1994 Mar;24(3):265-8.

2 Requena L, El-Shabrawi-Caelen L, Walsh SN, Segura S, Ziemer M, Hurt MA, et al. Intralymphatic histiocytosis. A clinicopathologic study of 16 cases. Am J Dermatopathol. 2009 Apr;31(2):140-51.

3 Saggar S, Lee B, Krivo J, Jacobson M, Krishnamurthy K. Intralymphatic histiocytosis associated with orthopedic implants. J Drugs Dermatol. 2011 Oct;10(10):1208-9.

4 Franz R, Andres C. Granulomatosa cheilitis and Melkersson-Rosenthal syndrome. Pathologist. 2014;35:17781.

5 Gómez-Sánchez ME, Azaña-Defez JM, Martínez-Martínez ML, López-Villaescusa MT. Intralymphatic Histiocytosis: A Report of 2 Cases, Actas Dermo-Sifiliográficas (English Edition), Volume 109, Issue 1, 2018, Pages e1-e5, ISSN 1578-2190.

6 Barba E, Colato C, Girolomoni G. Intralymphatic Histiocytosis: A case report and review of literature. J Cutan Pathol. 2015 Sep;42(9):593-9.

7 Requena L, El-Shabrawi-Caelen L, Walsh SN, Segura S, Ziemer M, Hurt MA, et al. Intralymphatic histiocytosis A clinicopathologic study of 16 cases. Am J Dermatopathol. 2009 Apr;31(2):140-51.

8 Demirkesen, C., Kran, T., Leblebici, C., Yücelten, D., Aksu, A. E. K., \& Mat, C. (2015, October). Intravascular/Intralymphatic Histiocytosis: A Report of 3 Cases.

9 R, F., \& C, A. (n.d.). Klinik und Poliklinik für Dermatologie und Allergologie am Biederstein, TU München, Biedersteinerstr. 29, 80802, München, Deutschland.

10 Sugano, T., Seike, M., Funasaka, Y. et al. Intralymphatic histiocytosis in a patient with lung adenocarcinoma treated with pembrolizumab: a case report. j. immunotherapy cancer 7, 59 (2019)

11 Barba, Eleonora, Colato, Chiara, and Girolomoni, Giampiero. "Intralymphatic Histiocytosis: A Case Report and Review of Literature." Journal of cutaneous pathology. 42.9 593-599. https://doi.org/10.1111/cup.12613. 


\section{Case Reports in Dermatology}

\begin{tabular}{l|l}
\hline Case Rep Dermatol 2021;13:1-6 \\
\hline DOI: 10.1159/000510128 & $\begin{array}{l}\text { (c) 2021 The Author(s). Published by S. Karger AG, Basel } \\
\text { www.karger.com/cde }\end{array}$ \\
\hline
\end{tabular}

Blackwell et al.: Intralymphatic Histiocytosis: An Unusual Presentation

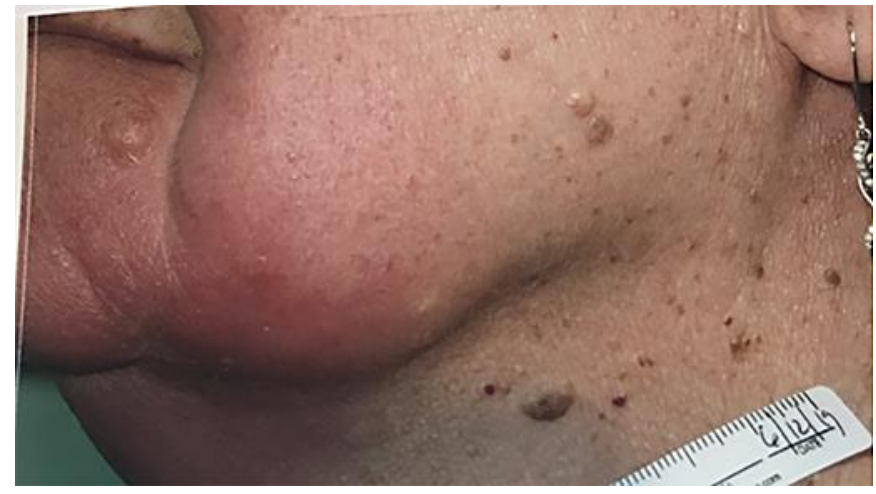

Fig. 1. Clinical presentation showing an ill-defined erythematous to violaceous plaque of the left lower face and chin.

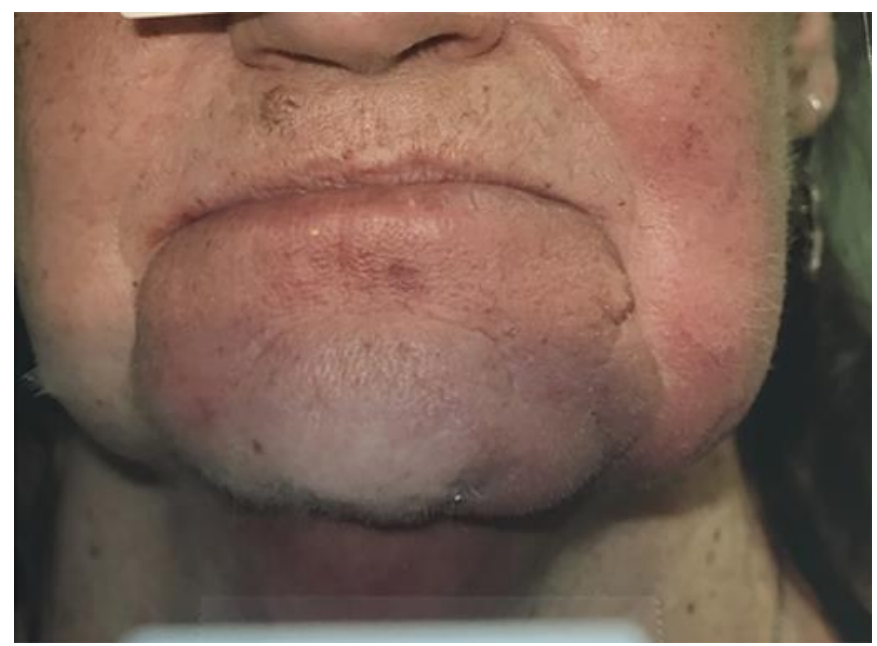

Fig. 2. Clinical presentation showing an ill-defined erythematous to violaceous plaque of the left lower face and chin. 

www.karger.com/cde

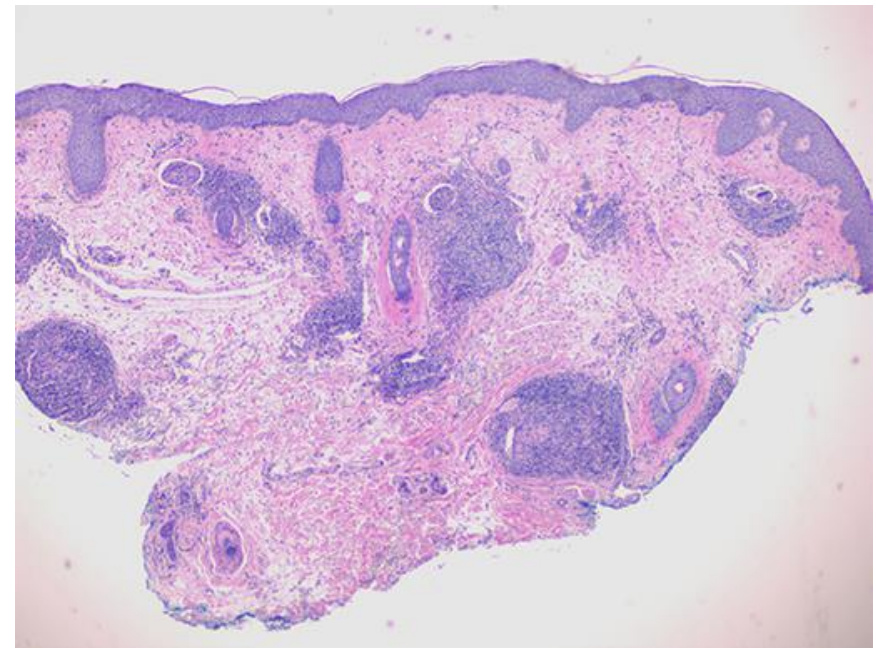

Fig. 3. Low power.

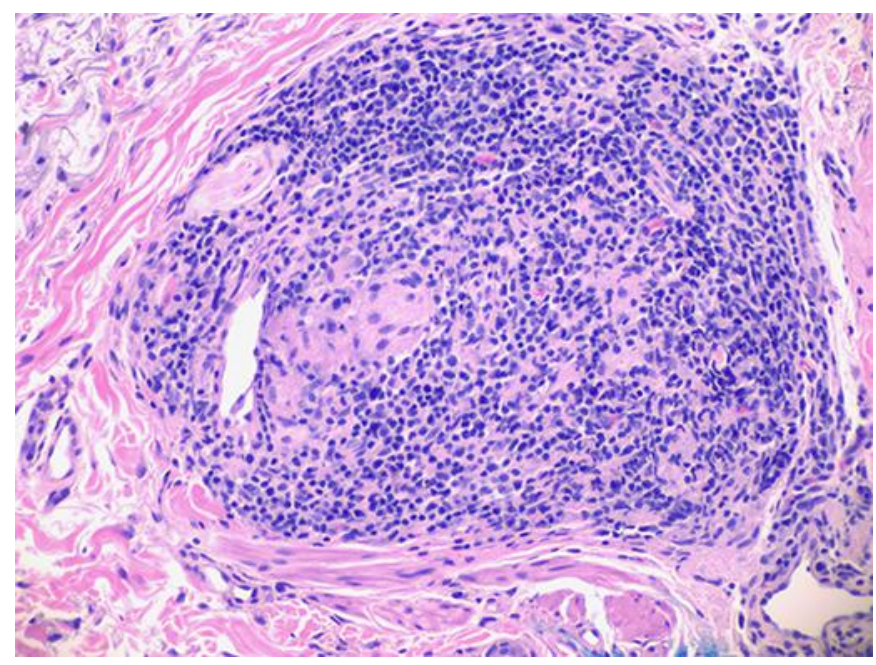

Fig. 4. Granulomatous and lymphoid aggregates. 